\title{
La percepción de amenaza física en la pareja y el bienestar en la juventud
}

\author{
Estefanía Mónaco Gerónimo \\ Dpto. Personalidad, Evaluación y Tratamientos Psicológicos. Universitat de València \\ estefania.monaco@uv.es \\ Victoria Ferrer Pérez \\ Dpto. Psicología Social. Universitat de les Illes Balears \\ victoria.ferrer@uib.es \\ Inmaculada Montoya Castilla \\ Dpto. Personalidad, Evaluación y Tratamientos Psicológicos. Universitat de València \\ inmaculada.montoya@uv.es
}

\begin{abstract}
RESUMEN
El presente trabajo estudia la percepción de amenaza hacia la seguridad y/o integridad física en las relaciones de pareja durante la juventud en relación con variables del bienestar personal y de pareja en la actualidad. Participaron 316 jóvenes entre 18 y 36 años $(M=23.61$; $D T=3.678), 72.47 \%$ se identificaron con el género femenino. Respecto a la orientación sexual, $64.87 \%$ fueron heterosexuales y $35.12 \%$ de otras orientaciones sexuales. Se evaluó la percepción de amenaza física en la pareja actual y relaciones pasadas (preguntas ad hoc), el bienestar con la pareja actual (SWRS), la satisfacción con la relación (RAS) y el bienestar subjetivo (SWLS y SPANE). La recogida de datos se realizó online, los datos fueron analizados mediante SPSS v.26, realizando estadísticos descriptivos, pruebas $t$, ANOVAs y correlaciones de Pearson. Entre los resultados obtenidos, destacamos que el $30.2 \%$ de las personas jóvenes manifiesta haber sentido amenazada su integridad física por lo menos en una ocasión en relaciones anteriores. En las personas bisexuales, este dato asciende al $44.6 \%$, siendo significativamente superior a las personas heterosexuales $(\mathrm{F}=5.34 ; \mathrm{p}=.001)$. De las personas con pareja actualmente, solo el 2.2\% informa percibir amenaza en alguna ocasión en dicha relación. La percepción de amenaza en relaciones anteriores se relaciona con mayor comunicación $(r=.18 ; \mathrm{p}=$ .017) y menores conflictos $(\mathrm{r}=-.17 ; \mathrm{p}=.025)$ en la relación de pareja actual, así como con mayor afecto negativo $(\mathrm{r}=0.19 ; \mathrm{p}=.004)$. Se discute la necesidad de estudiar la violencia en la pareja en todo tipo de relaciones de pareja durante la juventud, desarrollando herramientas para obtener indicadores precisos y sensibles sobre la prevalencia de diferentes tipos de violencias.
\end{abstract}

Palabras clave: violencia; pareja; juventud; agresión física; bienestar. 


\begin{abstract}
The present work studies the perception of threat to security and/or physical integrity in romantic partners during youth, related to personal and couple well-being. Participants were 316 young people between 18 and 36 years old $(\mathrm{M}=23.61$; $\mathrm{SD}=3.678) .72 .47 \%$ were female. Regarding sexual orientation, $64.87 \%$ were heterosexual and $35.12 \%$ of other sexual orientations. Perceived physical threat in current romantic relationship and past relationships (ad hoc questions), well-being (SWRS), relationship satisfaction (RAS) and subjective well-being (SWLS and SPANE) were assessed. Data collection was carried out online, the data were analyzed using SPSS v.26, performing descriptive statistics, t-tests, ANOVAs and Pearson correlations. Among the results obtained, we highlight that $30.2 \%$ of young people reported that they have felt their physical integrity threatened on at least once in previous relationships. Among bisexuals, this number rises to $44.6 \%$, being significantly higher than among heterosexuals $(F=5.34 ; p=.001)$. Of those with a current partner, only $2.2 \%$ report perceiving threat on some occasion in this romantic relationship. Perceived threat in previous relationships is related to greater communication $(\mathrm{r}=.18 ; \mathrm{p}=.017)$ and less conflict $(\mathrm{r}=-.17 ; \mathrm{p}=.025)$ in the current romantic relationship, as well as greater negative affect $(r=.19 ; \mathrm{p}=.004)$. The need to study intimate partner violence in all types of romantic relationships during youth is discussed, developing tools to obtain accurate and sensitive indicators on the prevalence of different types of violence.
\end{abstract}

Keywords: violence; couple; youth; physical aggression; well-being.

Este trabajo se ha realizado gracias a la beca predoctoral Atracció de Talent de la Universitat de València (UV-INV_PREDOC17F1-540334) y sus ayudas a la movilidad. 


\section{INTRODUCCIÓN}

La violencia es una forma de interacción entre seres vivos caracterizada por el ejercicio de poder o de coacción sobre otra persona, que deriva en daños físicos, psicológicos o morales para la persona victimizada (Farr, 2021). Existen muchos y muy diferentes tipos de violencia, algunos más visibilizados como la violencia psicológica, la física o la sexual, otros menos nombrados como la violencia económica, la violencia simbólica o la violencia institucional, entre otras.

Un tipo de violencia es aquella que ocurre contra las mujeres en la pareja, también denominada en el ordenamiento jurídico español como "violencia de género" o "violencia machista" (Gimeno \& Barrientos, 2009). Esta violencia asienta sus raíces en la construcción social patriarcal, la cual afecta a todas las personas fuera de la norma de género tradicionalmente heterosexual y patriarcal (Bosch-Fiol et al., 2006). La violencia contra las mujeres es un problema social y de salud pública de primer orden en la actualidad (Morales, 2021).

Dada su elevada prevalencia, tanto en España como a nivel mundial, no es de extrañar que la población más investigada y atendida en cuanto a violencia en la pareja han sido las mujeres heterosexuales, siendo menos estudiadas otras personas también afectadas por la violencia basada en el género que ocurre en las relaciones de pareja: personas transgénero o no binarias, las personas con orientaciones sexuales diferentes a la heterosexual, entre otras (Callan et al., 2021; DeKeseredy et al., 2019). Ésta es una realidad que es necesario atender, dado que es una grave amenaza tanto para la salud como para el bienestar subjetivo (Botello-Peñaloza \& Guerrero-Rincón, 2018; Callan et al., 2021).

La violencia impacta de manera grave tanto en el bienestar de la relación de pareja, como en el bienestar personal (Ulloa et al., 2015; Kaura \& Lohman, 2007). Respecto a la salud mental, la prevalencia de sintomatología depresiva y otros síntomas psicopatológicos es elevada en las personas que sufren violencia (Ahmadabadi, 2020; Murillo et al., 2004). Ser víctima de violencia está fuertemente relacionado con la afectividad negativa (Santandreu \& Ferrer, 2014), definida como una variedad de estados de ánimo, que incluyen la ira, la culpa, el temor y el nerviosismo (Watson et al., 1988). Uno de los sentimientos más frecuentes es el sentimiento de culpa (Santandreu \& Ferrer, 2014). Respecto a la ira, las personas que sufren violencia parecen tener tendencia a suprimirla (Santandreu \& Ferrer, 2014).

A pesar de sus preocupantes consecuencias, la violencia en la pareja es una realidad que tiende a invisibilizarse, siendo complicado obtener datos precisos sobre la prevalencia real de la violencia en las parejas (Botello-Peñaloza \& Guerrero-Rincón, 2018; FerrerPérez \& Bosch-Fiol, 2014). Esto ocurre por diferentes motivos (Observatorio de Salud de la Mujer, 2015): (1) se desarrolla en un espacio íntimo y privado, como es la relación de pareja, por lo que es difícil su observación; (2) únicamente es posible obtener los datos a través de la información subjetiva que nos proporcionan las personas, sujetas a posibles distorsiones o sesgos (p. e. dificultad de reconocer la violencia en sus formas más sutiles, normalización o aceptación social de la violencia); (3) intentos de ocultar externamente que se sufre violencia en la pareja, un tema que generalmente ha sido tabú para la 
sociedad, y el cual genera fuertes sentimientos de vergüenza, culpa e incomprensión por parte de las víctimas.

La violencia física fue una de las primeras violencias en ser estudiadas, al ser la más accesible de observar externamente (Botello-Peñaloza \& Guerrero-Rincón, 2018). Realizando una revisión de algunos de los instrumentos utilizados para evaluar violencia física (Observatorio de Salud de la Mujer, 2015), se encuentra que sus ítems preguntan por la experimentación de comportamientos violentos, con qué frecuencia y a qué nivel de intensidad han sucedido. Sin embargo, son más difíciles de captar aquellos casos en los que el acto agresivo no se llega a producir, ni tampoco existe una amenaza directa del mismo, pero la víctima se siente amenazada físicamente en la relación. Esta percepción de amenaza podría encontrarse en un terreno intermedio entre la violencia física y la violencia psicológica.

El objetivo del presente trabajo fue estudiar la percepción que tienen las personas jóvenes de haber sentido amenazada su seguridad o su integridad física en sus relaciones de pareja anteriores y en relación de pareja actual si la tienen, analizando cómo se relaciona esta experiencia de inseguridad con el bienestar personal y de pareja.

\section{MÉTODO}

\section{Participantes}

Participaron 316 jóvenes entre 18 y 36 años $(\mathrm{M}=23.61$; DT = 3.678). Respecto al género, $229(72.47 \%)$ se identificaron con el género femenino, $85(26.90 \%)$ con el masculino, y $2(0.63 \%)$ con el género no binario. Sobre la orientación sexual, 205 personas (64.87\%) informaron ser heterosexuales y $111(35.12 \%)$ no heterosexuales, concretamente $90(28.48 \%)$ bisexuales, $16(5.06 \%)$ homosexuales y $6(1.90 \%)$ de otras orientaciones sexuales. De las personas participantes, $183(57.91 \%)$ tienen pareja, 106 (33.54\%) no la tienen, y 27 (8.54\%) indican tener otro tipo de relación sexoafectiva diferente.

\section{Variables e instrumentos}

Variables sociodemográficas. Edad, género, orientación sexual, relación de pareja en la actualidad y género de la pareja actual.

Percepción de amenaza física en relaciones de pareja. Se evaluó mediante dos preguntas ad hoc ("En tu relación de pareja actual/en tus relaciones de pareja anteriores, ¿sientes o has sentido amenazada tu seguridad o tu integridad física?”) con una escala de respuesta tipo Likert de cuatro puntos ( 1 = Nunca; 4 = Habitualmente).

Bienestar subjetivo. Se evaluó la satisfacción con la vida mediante la Satisfaction With Life Scale (SWLS; Diener et al., 1985), un instrumento que cuenta con 5 ítems de 7 alternativas de respuesta $(1=$ Completamente en desacuerdo; $7=$ Completamente de acuerdo) para medir la valoración positiva de las condiciones de la propia vida. También se utilizó la Escala de Experiencias Positivas y Negativas (SPANE; Diener et al., 2009), 
compuesta por 12 ítems con una escala tipo Likert de 5 puntos $(1=$ Nunca; $5=$ Siempre $)$, para medir la afectividad positiva y negativa.

Bienestar en la pareja. Se utilizó en primer lugar el instrumento Subjective WellBeing in Couple Relationship Scale (SWCR; Urbano-Contreras et al., 2018), el cual cuenta con 19 ítems con respuestas en una escala tipo Likert de 4 puntos ( 1 = Totalmente en desacuerdo; 4 = Totalmente de acuerdo). Concretamente se utilizaron los factores "Grado de comunicación" y "Frecuencia de conflictos". Además, para medir la satisfacción con la pareja se utilizó el Relationship Assessment Scale (RAS; Hendrick, Dicke, et al., 1998), compuesto por 7 ítems con una escala Likert que oscila entre 1 (Totalmente en desacuerdo) y 5 (Totalmente de acuerdo).

\section{Procedimiento}

Las personas participaron en esta investigación de manera online, mediante un enlace a un cuestionario de la plataforma Limesurvey. Previamente a su participación, fueron informadas de los objetivos del estudio y dieron su consentimiento informado. La muestra fue de conveniencia, realizándose la captación a través de la difusión del enlace mediante redes sociales e Internet. Se respetaron en todo momento las normativas éticas propuestas en la Declaración de Helsinki (World Medical Association, 2013) para la investigación en humanos.

\section{Análisis de datos}

Los datos se analizaron mediante el paquete estadístico SPSS Versión 26.0. Se realizaron análisis descriptivos para estudiar las características de la muestra, ANOVAs y pruebas $t$ para estudiar las diferencias significativas según género y orientación sexual, y correlaciones de Pearson para comprender la relación entre las variables estudiadas.

\section{RESULTADOS}

\section{Frecuencias de percepción de amenaza en relación actual y anteriores}

Si estudiamos la percepción de amenaza hacia la integridad y/o seguridad física en la relación de pareja actual (Tabla 1), observamos que únicamente el 2.2\% las personas que actualmente tienen pareja $(n=183)$ dice sentir dicha amenaza en alguna ocasión. El resto de personas $(97.8 \%)$ afirma no sentirse amenazada actualmente.

Los datos cambian notablemente cuando preguntamos por la percepción de amenaza en relaciones de pareja anteriores (Tabla 1). Entonces observamos que el $69.7 \%$ dice no haberla sufrido nunca, el $14.6 \%$ en alguna ocasión, el $11.8 \%$ en más de una ocasión, y el $3.8 \%$ habitualmente.

Si analizamos a estas personas según su orientación sexual (Tabla 1), vemos que el $77.6 \%$ de las personas heterosexuales dice no haber sentido amenazada nunca su seguridad en el pasado. En personas bisexuales el porcentaje parece preocupante: el $55.3 \%$ afirman no haberse sentido nunca amenazadas, lo cual significa que el $44.7 \%$ de 
personas bisexuales sí ha sentido amenazada su seguridad física en relaciones anteriores por lo menos una vez.

Tabla 1. Percepción de amenaza a la seguridad y/o integridad física según la orientación sexual

\begin{tabular}{|c|c|c|c|c|c|c|c|c|}
\hline & \multicolumn{4}{|c|}{ En la relación de pareja actual } & \multicolumn{4}{|c|}{ En relaciones de pareja anteriores } \\
\hline & $\begin{array}{l}\text { Nunca } \\
\text { n (\%) }\end{array}$ & $\begin{array}{c}\text { En alguna } \\
\text { ocasión } \\
\mathrm{n}(\%)\end{array}$ & $\begin{array}{c}\text { En más de } \\
\text { una } \\
\text { ocasión } \\
\mathrm{n}(\%)\end{array}$ & $\begin{array}{c}\text { Habitualm } \\
\text { ente } \\
\text { n (\%) }\end{array}$ & $\begin{array}{l}\text { Nunca } \\
\text { n (\%) }\end{array}$ & $\begin{array}{c}\text { En alguna } \\
\text { ocasión } \\
\text { n }(\%)\end{array}$ & $\begin{array}{c}\text { En más de una } \\
\text { ocasión } \\
\text { n }(\%)\end{array}$ & $\begin{array}{c}\text { Habitualm } \\
\text { ente } \\
\text { n (\%) }\end{array}$ \\
\hline Heterosexual & $117(97.5 \%)$ & $3(2.5 \%)$ & $0(0 \%)$ & $0(0 \%)$ & $142(77.6 \%)$ & $21(11.5 \%)$ & $16(8.7 \%)$ & $4(2.2 \%)$ \\
\hline Homosexual & $8(100 \%)$ & $0(0 \%)$ & $0(0 \%)$ & $0(0 \%)$ & $9(64.3 \%)$ & $2(14.3 \%)$ & $3(21.4 \%)$ & $0(0 \%)$ \\
\hline Bisexual & $50(98 \%)$ & $1(2 \%)$ & $0(0 \%)$ & $0(0 \%)$ & $47(55.3 \%)$ & $16(18.8 \%)$ & $15(17.6 \%)$ & $7(8.2 \%)$ \\
\hline Otra & $3(100 \%)$ & $0(0 \%)$ & $0(0 \%)$ & $0(0 \%)$ & $2(40 \%)$ & $3(60 \%)$ & $0(0 \%)$ & $0(0 \%)$ \\
\hline Total & $178(97.8 \%)$ & $4(2.2 \%)$ & $0(0 \%)$ & $0(0 \%)$ & $200(69.7 \%)$ & $42(14.6 \%)$ & $34(11.8 \%)$ & $11(3.8 \%)$ \\
\hline
\end{tabular}

Si analizamos la frecuencia de amenaza en relaciones anteriores según la identidad de género, observamos los siguientes datos en las mujeres: $64.3 \%(n=137)$ dice no haber sentido nunca amenaza, $15.5 \%(\mathrm{n}=33)$ en una ocasión, $15.5 \%(\mathrm{n}=33)$ en más de una ocasión y $4.7 \%(n=10)$ habitualmente. En cuanto a los hombres, $87.5 \%(n=63)$ dice no haber sentido nunca amenaza, 9.7\% $(\mathrm{n}=7)$ en una ocasión, $1.4 \%(\mathrm{n}=1)$ en más de una ocasión y $1.4 \%(\mathrm{n}=1)$ habitualmente. Las dos personas de género no binario afirman haber sentido amenaza física en relaciones anteriores en una ocasión.

\section{Estadísticos descriptivos y diferencias según variables sociodemográficas}

En la Tabla 2 se pueden observar las medias y desviaciones típicas de las variables estudiadas tanto en función del género como de la orientación sexual.

Como se observa en la Tabla 2, la media de percepción de amenaza en relaciones anteriores es superior: (1) en mujeres frente a hombres, siendo esta diferencia significativa $(t=3.88 ; \mathrm{p}=.000)$, y (2) en personas no heterosexuales frente a heterosexuales, siendo también significativa la diferencia $(t=-3.60 ; \mathrm{p}=.000)$.

Tabla 2. Estadísticos descriptivos atendiendo al género y a la orientación sexual

\begin{tabular}{|c|c|c|c|c|c|c|c|c|}
\hline & \multicolumn{2}{|c|}{$\begin{array}{c}\text { Mujeres } \\
\text { Con pareja: } \mathrm{n}= \\
139 \\
\text { Sin pareja: } \mathrm{n}=90 \\
\text { Total mujeres: } \mathrm{n}= \\
229\end{array}$} & \multicolumn{2}{|c|}{$\begin{array}{c}\text { Hombres } \\
\text { Con pareja: } n=42 \\
\text { Sin pareja: } n=43 \\
\text { Total hombres: } n \\
=85\end{array}$} & \multicolumn{2}{|c|}{$\begin{array}{c}\text { Heterosexuales } \\
\text { Con pareja: } \mathrm{n}= \\
121 \\
\text { Sin pareja: } \mathrm{n}=84 \\
\text { Total hetero: } \mathrm{n}= \\
205\end{array}$} & \multicolumn{2}{|c|}{$\begin{array}{c}\text { No heterosexuales } \\
\text { Con pareja: } n=62 \\
\text { Sin pareja: } n=49 \\
\text { Total no hetero: } n= \\
111\end{array}$} \\
\hline & Rango & M (DT) & Rango & M (DT) & Rango & M (DT) & Rango & M (DT) \\
\hline $\begin{array}{l}\text { Amenaza relaciones } \\
\text { antes }\end{array}$ & 3.00 & $\begin{array}{l}1.61 \\
(.91)\end{array}$ & 3 & $\begin{array}{l}1.17 \\
(.50)\end{array}$ & 3 & $\begin{array}{l}1.36 \\
(.73)\end{array}$ & 3 & $\begin{array}{l}1.75 \\
(.97)\end{array}$ \\
\hline $\begin{array}{l}\text { Amenaza relación } \\
\text { actual }\end{array}$ & 1.00 & $\begin{array}{l}1.02 \\
(.15)\end{array}$ & 1 & $\begin{array}{l}1.02 \\
(.16)\end{array}$ & 1 & $\begin{array}{l}1.03 \\
(.16)\end{array}$ & 1 & $\begin{array}{l}1.02 \\
(.13)\end{array}$ \\
\hline
\end{tabular}




\begin{tabular}{|c|c|c|c|c|c|c|c|c|}
\hline $\begin{array}{l}\text { Comunicación } \\
\text { pareja }\end{array}$ & 2.00 & $\begin{array}{l}3.65 \\
(.49)\end{array}$ & 2.00 & $\begin{array}{l}3.38 \\
(.59)\end{array}$ & 2.00 & $\begin{array}{l}3.57 \\
(.53)\end{array}$ & 1.50 & $\begin{array}{l}3.63 \\
(.52)\end{array}$ \\
\hline Conflictos pareja & 2.50 & $\begin{array}{l}2.21 \\
(.70)\end{array}$ & 2.50 & $\begin{array}{l}2.38 \\
(.75)\end{array}$ & 2.50 & $\begin{array}{l}2.26 \\
(.70)\end{array}$ & 2.50 & $\begin{array}{l}2.19 \\
(.76)\end{array}$ \\
\hline $\begin{array}{l}\text { Satisfacción } \\
\text { relación }\end{array}$ & 2.29 & $\begin{array}{l}4.30 \\
(.50)\end{array}$ & 2.29 & $\begin{array}{l}4.19 \\
(.55)\end{array}$ & 2.29 & $\begin{array}{l}4.31 \\
(.50)\end{array}$ & 2.29 & $\begin{array}{l}4.21 \\
(.53)\end{array}$ \\
\hline $\begin{array}{l}\text { Satisfacción con la } \\
\text { vida }\end{array}$ & 6.00 & $\begin{array}{c}4.83 \\
(1.21)\end{array}$ & 5.80 & $\begin{array}{c}4.61 \\
(1.30)\end{array}$ & 5.80 & $\begin{array}{l}4.95 \\
(1.16)\end{array}$ & 6.00 & $\begin{array}{c}4.44 \\
(1.30)\end{array}$ \\
\hline Afecto positivo & 3.33 & $\begin{array}{l}3.61 \\
(.67)\end{array}$ & 3.50 & $\begin{array}{l}3.45 \\
(.72)\end{array}$ & 3.17 & $\begin{array}{l}3.63 \\
(.65)\end{array}$ & 3.33 & $\begin{array}{l}3.43 \\
(.73)\end{array}$ \\
\hline Afecto negativo & 3.83 & $\begin{array}{l}2.59 \\
(.66)\end{array}$ & 2.67 & $\begin{array}{l}2.51 \\
(.57)\end{array}$ & 3.83 & $\begin{array}{l}2.48 \\
(.61)\end{array}$ & 2.83 & $\begin{array}{l}2.76 \\
(.66)\end{array}$ \\
\hline
\end{tabular}

Tras realizar un ANOVA con las diferentes orientaciones sexuales (heterosexual, homosexual, bisexual y otra), observamos que la diferencia significativa en percepción de amenaza en relaciones anteriores $(F=5.34 ; \mathrm{p}=.001)$ se encuentra entre las personas heterosexuales $(\mathrm{M}=1.36$; DT $=.73)$ y las bisexuales $(\mathrm{M}=1.79$; $\mathrm{DT}=1.01)$, siendo estas últimas las que han sufrido mayor percepción de amenaza.

Esta muestra bisexual estuvo formada por 79 mujeres y 10 hombres. De esas 79 mujeres, 59 tienen pareja actualmente: 35 tienen una relación con un hombre y 19 con una mujer o con una persona de género no binario. Tras realizar una prueba $t$ entre estos dos grupos, observamos que las mujeres bisexuales que actualmente tienen una relación con un hombre puntúan significativamente más alto $(\mathrm{M}=2.23$; $\mathrm{DT}=1.19)$ en amenaza en relaciones pasadas $(t=.39 ; \mathrm{p}=.000)$ que aquellas que están en relaciones no heterosexuales $(\mathrm{M}=1.26$; DT $=.45)$.

\section{Relaciones entre las variables estudiadas}

Como se observa en la Tabla 3, la percepción de amenaza en la relación de pareja actual correlaciona de forma significativa y negativa con la comunicación con la pareja, con la satisfacción con la relación y con la satisfacción con la vida.

La percepción de amenaza en relaciones anteriores correlaciona de forma significativa y positiva con la comunicación con la pareja actual y con el afecto negativo en el presente, y negativamente con los conflictos con la pareja actual (Tabla 3 ).

Tabla 3. Correlaciones bivariadas entre las variables

\begin{tabular}{|c|c|c|c|c|c|c|c|}
\hline & 1 & 2 & 3 & 4 & 5 & 6 & 7 \\
\hline 1. Amenaza relaciones antes & 1 & & & & & & \\
\hline 2. Amenaza relación actual & .12 & 1 & & & & & \\
\hline 3. Comunicación pareja & $.18^{*}$ & $-.17^{*}$ & 1 & & & & \\
\hline 4. Conflictos pareja & $-.17^{*}$ & .08 & $-.32^{* *}$ & 1 & & & \\
\hline 5. Satisfacción relación & .01 & $-.15^{*}$ & $.56^{* *}$ & $-.40^{* *}$ & 1 & & \\
\hline 6. Satisfacción con la vida & -.08 & $-.16^{*}$ & $.37^{* *}$ & -.09 & $.32^{* *}$ & 1 & \\
\hline 7. Afecto positivo & -.10 & -.18 & $.42^{* *}$ & $-.24^{* *}$ & $.38^{* *}$ & $.55^{* *}$ & 1 \\
\hline
\end{tabular}




\begin{tabular}{llllllll}
\hline 8. Afecto negativo & $.19^{* * *}$ & .11 & $-.25^{* *}$ & $.20^{*}$ & $-.25^{* *}$ & $-.42^{* *}$ & $-.63^{* *}$ \\
\hline${ }^{*} \mathrm{p}<.05 ;{ }^{* *} \mathrm{p}<.01$ & & & & & & &
\end{tabular}

Si estudiamos la correlación entre la percepción de amenaza y las diferentes emociones medidas por el SPANE (felicidad, tristeza, miedo, alegría, enfado y satisfacción), observamos que sentir amenaza en la relación actual correlaciona de forma significativa y negativa con la felicidad $(\mathrm{r}=-.19 ; \mathrm{p}=.016)$, la alegría $(\mathrm{r}=-.20 ; \mathrm{p}=.014)$ y la satisfacción $(\mathrm{r}=-.17 ; \mathrm{p}=.035)$, y de forma positiva con la tristeza $(\mathrm{r}=.17 ; \mathrm{p}=.042)$. Haber sentido amenaza en relaciones anteriores se relaciona con sentir miedo en la actualidad $(\mathrm{r}=.17 ; \mathrm{p}=.010)$. No se encuentran correlaciones con la emoción de enfado.

Del resto de variables estudiadas, destacamos que algunas de las correlaciones más fuertes son las encontradas respecto a la comunicación con la pareja y la satisfacción con la relación $(\mathrm{r}=.56 ; \mathrm{p}=.000)$, así como con el afecto positivo $(\mathrm{r}=.42 ; \mathrm{p}=.000)$. La correlación también es fuerte entre la satisfacción con la relación de pareja y el afecto positivo $(\mathrm{r}=.38 ; \mathrm{p}=.000)$. Todas las correlaciones pueden apreciarse detalladamente en la Tabla 4.

\section{DISCUSIÓN}

El objetivo del presente trabajo fue estudiar si las personas jóvenes entre 18 y 36 años perciben amenazada su seguridad o integridad física en sus relaciones de pareja, tanto en relaciones anteriores como en su relación actual si la tienen. Además, se analizó la relación de dicha percepción de amenaza con el bienestar personal y de pareja.

Algunas de las conclusiones principales de nuestros resultados son que el $22.4 \%$ de las personas heterosexuales ha sentido amenaza en relaciones anteriores por lo menos una vez, mientras que el $46.8 \%$ de las personas de otras orientaciones sexuales diferente a la heterosexual informa haber sufrido dicha amenaza en el pasado. Estos datos confirman la idea de que la violencia en las relaciones de pareja no ocurre únicamente en las relaciones heterosexuales normativas de hombre y mujer heterosexuales (Callan et al., 2021), sino en todo tipo de parejas. Respecto al género, el $35.7 \%$ de las mujeres afirma haber sentido amenazada su seguridad física en relaciones anteriores por lo menos una vez, siendo este porcentaje del $12.5 \%$ para los hombres. Concretamente, según nuestros resultados, las mujeres bisexuales parecen ser el grupo con mayor vulnerabilidad de sufrir amenazas hacia su seguridad y/o integridad física, especialmente aquellas en una relación con una persona de género masculino.

Otro dato destacable es la cifra notablemente baja de personas que informan sentir amenazada su seguridad o integridad física en su relación de pareja actual (2.2\%). La primera conclusión de este dato podría ser que la juventud en la actualidad está aprendiendo a reconocer los signos de violencia, incluso aquellos más sutiles, por lo que no toleran mantenerse en relaciones donde perciban amenazada su seguridad o integridad física. Una interpretación menos optimista de este resultado apoyaría la teoría de la invisibilización de la violencia en la pareja y la dificultad de obtener datos realistas sobre la misma. Es posible que los resultados estén minimizados, si las personas participantes 
han sesgado sus respuestas por la propia vergüenza, sensación de incomprensión o culpa que genera a las víctimas estar sufriendo esta situación en su pareja (Observatorio de Salud de la Mujer, 2015).

Por una parte, observamos que sentirse amenazada en la relación actual correlaciona negativamente con la comunicación con la pareja y la satisfacción con la relación, lo cual es coherente con la literatura (Ulloa et al., 2015). Por otra parte, vemos que haber sentido amenaza en relaciones anteriores correlaciona con una mayor comunicación y un menor número de conflictos con la pareja actual. Esta relación, aunque no es causal sino correlacional, nos lleva a diferentes inferencias. En primer lugar, podríamos pensar que las personas que han sufrido situaciones potencialmente violentas están aprendiendo a construir en la actualidad relaciones basadas en el diálogo, para evitar que dichas situaciones se repitan. Otra interpretación podría ser que las potenciales víctimas de violencia tienen un estilo más sumiso de comunicación con sus parejas, evitando en lo posible los conflictos (Kaura \& Lohman, 2007).

Por último, si nos centramos en el bienestar personal, se observa que sentirse amenazada en la actualidad se relaciona con menor satisfacción con la vida y con una tendencia hacia la depresión, ya que se asocia con un bajo afecto positivo (poca felicidad, alegría y satisfacción) y un alto afecto negativo (tristeza). Haber sentido amenaza en relaciones anteriores se relaciona actualmente con una tendencia aparentemente más ansiosa, relacionándose únicamente con el afecto negativo, y particularmente con la emoción de miedo. Estos resultados van en la línea de lo encontrado por Santandreu y Ferrer (2014), incluso la ausencia de relación en nuestros resultados con la emoción de ira, la cual parece tener tendencia a ser suprimida en las personas víctimas de violencia.

El presente trabajo no está libre de limitaciones. Una de las principales limitaciones es la desproporción entre hombres y mujeres, así como entre personas de diferentes orientaciones sexuales. Además, la medida utilizada para evaluar la percepción de amenaza es ad hoc, con su respectiva carencia de validación. Por ello, sería imprescindible en el futuro continuar investigando en esta línea, ampliando la muestra de género masculino, de personas no binarias y de personas homosexuales y de otras orientaciones sexuales. También sería necesario ampliar el número de preguntas sobre la percepción de amenaza para comprender esta variable en mayor detalle, utilizando también otros cuestionarios previamente validados para compararlos con nuestra variable y poder evaluar sus propiedades psicométricas. Por último, sería de interés evaluar otras formas de violencia, como la psicológica o la sexual.

A pesar de sus limitaciones, consideramos que el presente trabajo es una forma de generar conciencia ante la necesidad de atender un fenómeno tan complejo como es la violencia en la pareja en todo tipo de relaciones, y no únicamente en las relaciones heterosexuales normativas (DeKeseredy et al., 2019). Necesitamos desarrollar nuevos instrumentos en el contexto sociocultural de nuestro país (Observatorio de Salud de la Mujer, 2015), que sean sensibles a todo tipo de realidades personales, incluyendo aquellas que experimentan las personas no-normativas. Con este trabajo también pretendemos remarcar la importante necesidad de educar a la juventud en el establecimiento y mantenimiento de relaciones afectivas sanas, igualitarias y libres de violencia, como una 
forma de promover el bienestar de esta población (Ahmadabadi et al., 2020; Callan et al., 2021).

\section{REFERENCIAS}

Ahmadabadi, Z., Najman, J. M., Williams, G. M., Clavarino, A. M., d'Abbs, P., \& Tran, N. (2020). Intimate partner violence and subsequent depression and anxiety disorders. Social Psychiatry and Psychiatric Epidemiology, 1-10. https://doi.org/10.1007/s00127-019-01828-1

Botello-Peñaloza, H. A., \& Guerrero-Rincón, I. (2018). Incidencia de la violencia física en la población LGBT en Ecuador. Civilizar: Ciencias Sociales y Humanas, 18(35), 129-138. https://doi.org/10.22518/usergioa/jour/ccsh/2018.2/a10

Callan, A., Corbally, M., \& McElvaney, R. (2021). A scoping review of intimate partner violence as it relates to the experiences of gay and bisexual men. Trauma, Violence, \& Abuse, 22(2), 233-248. https://doi.org/10.1177\%2F1524838020970898

Morales Carrero, J. A. (2021). Violencia de género. Un problema de salud pública en tiempos de pandemia. Interamerican Journal of Medicine and Health, 4-18. https://doi.org/10.31005/iajmh.v4i.176

DeKeseredy, W. S., Nolan, J., Hall-Sanchez, A., \& Messinger, A. M. (2019). Intimate partner violence victimization among heterosexual, gay, lesbian and bisexual college students: The role of pro-abuse peer support. Journal of Aggression, Maltreatment \& Trauma, 28(9), 1057-1068. https://doi.org/10.1080/10926771.2018.1551820

Diener, E. D., Emmons, R. A., Larsen, R. J., \& Griffin, S. (1985). The satisfaction with life scale. Journal of Personality Assessment, 49(1), 71-75. https://doi.org/10.1207/s15327752jpa4901_13

Diener, E., Wirtz, D., Biswas-Diener, R., Tov, W., Kim-Prieto, C., Choi, D. W., \& Oishi, S. (2009). New measures of well-being. En Diener E. (Ed.), Assessing Well-being (pp. 247-266). Springer. https://doi.org/10.1007/978-90-481-2354-4_12

Farr, P. (2021). Crashing bodies: towards an intersectional assemblage theory of domestic violence. Feminist Theory. https://doi.org/10.1177\%2F14647001211014764

Ferrer-Pérez, V. A., \& Bosch-Fiol, E. (2014). Gender violence as a social problem in Spain: Attitudes and acceptability. Sex Roles, 70, 506-521. https://doi.org/10.1007/s11199-013-0322-Z

Bosch-Fiol, E., Ferrer-Pérez, V. A., \& Alzamora-Mir, A. (2006). El laberinto patriarcal: reflexiones teórico-prácticas sobre la violencia contra las mujeres. Anthropos.

Gimeno Reinoso, B., \& Barrientos Silva, V. (2009). Violencia de género versus violencia doméstica: la importancia de la especificidad. Revista Venezolana de Estudios de la Mujer, 14(32), 27-42. 
Hendrick, S., Dicke, A., \& Hendrick, C. (1998). The Relationship Assessment Scale. Journal of Social and Personal Relationships (15), 137-142. https://doi.org/10.1177\%2F0265407598151009

Kaura, S. A., \& Lohman, B. J. (2007). Dating violence victimization, relationship satisfaction, mental health problems, and acceptability of violence: A comparison of men and women. Journal of Family Violence, 22(6), 367-381. https://doi.org/10.1007/s10896-007-9092-0

Murillo, F. H., Vives, C. C., \& Rubio, M. (2004). El conflicto de pareja como variable asociada a la violencia de género contra la mujer: consecuencias sobre la salud sexual y mental. Revista de Psicopatología y Psicología Clínica, 9(1), 49-64. https://doi.org/10.5944/rppc.vol.9.num.1.2004.3967

Observatorio de Salud de la Mujer (2015). Catálogo de instrumentos para el cribado y frecuencia del maltrato físico, psicológico y sexual [Archivo PDF]. https://www.mscbs.gob.es/organizacion/sns/planCalidadSNS/pdf/equidad/genero_v g_01.pdf

Santandreu, M., \& Ferrer, V. A. (2014). Análisis de la emotividad negativa en mujeres víctimas de violencia de pareja: la culpa y la ira. Revista de Psicopatología y $\begin{array}{lll}\text { Psicología } \quad \text { Clínica, } & 19(2), & 129-140 .\end{array}$ https://doi.org/10.5944/rppc.vol.19.num.2.2014.13063

Ulloa, E. C., \& Hammett, J. F. (2015). Temporal changes in intimate partner violence and relationship satisfaction. Journal of Family Violence, 30(8), 1093-1102. https://doi.org/10.1007/s10896-015-9744-4

Urbano-Contreras, A., Martínez-González, R. A., \& Iglesias-Garcia, M. T. (2018). Validation of the Subjective Well-Being in Couple Relationship Scale (SWCR). Marriage and Family Review, 54(6), 598-615. https://doi.org/10.1080/01494929.2018.1435435

Watson, D., Clark, L. A., \& Tellegen, A. (1988). Development and validation of brief measures of positive and negative affect: the PANAS scales. Journal of Personality and Social Psychology, 54(6), 1063-107. https://doi.org/10.1037/00223514.54.6.1063

World Medical Association (2013). World Medical Association Declaration of Helsinki: Ethical principles for medical research involving human subjects. The Journal of the American Medical Association, 310, 2191-2194. 
